\title{
Écrire la guerre civile algérienne : la mise en place d'un nouveau pacte de lecture dans Le Serment des barbares de Boualem Sansal
}

\author{
Lisa ROMAIN \\ Université Charles de Gaulle - Lille 3 \\ France
}

Résumé : Durant la décennie noire les romanciers francophones algériens ont été confrontés à des dysfonctionnements dans la réception de leurs œuvres. Pour diverses raisons, le sens de ces fictions a parfois été déplacé. Boualem Sansal, dont le premier roman paraît en 1999, met au point des stratégies littéraires originales pour contourner les écueils qu'il a pu observer tout au long des années 90. Il pose les jalons d'un pacte de lecture fondé sur l'éthique et la responsabilité. L'auteur semble ainsi indiquer que la dénonciation de la violence et de ses facteurs doit pour être efficace impliquer le lecteur comme partenaire actif.

\begin{abstract}
During the Black Decade, Algerian French speaking writers were confronted with several issues in the way their novels were received. The meaning of these novels has often been misrepresented. Boualem Sansal, whose first novel was published in 1999, develops original generic and enunciative strategies to bypass the obstacles that he noticed during these troubled years. He lays the foundations for a new deal with his readers, based on ethics and responsibility. The author aims to show that the denunciation of violence and its root causes can only make a significant impact if the reader is involved as an active partner.
\end{abstract}

Mots-clés : Algérie, guerre civile, réception, énonciation, responsabilisation.

Keywords : Algeria, civil war, reception, enunciation, accountability.

\section{Une réception paralysée}

L'extrême violence de la guerre civile qui a meurtri l'Algérie tout au long des années $90^{1}$ a "[bouleversé] le paysage littéraire et intellectuel » (Moura 1999, 119). Écrivains confirmés ou débutants ont tenté d'exorciser l'horreur, principalement dans des fictions souvent identifiées comme des «écritures de l'urgence » (Boualit 1999, 36-37). Ces romans, tout particulièrement lorsqu'ils étaient écrits en français, ont achoppé sur des problèmes de réception multiples.

\section{a) La réception algérienne}

Les intrigues du corpus des grands auteurs algériens des années 90 remontent bien souvent aux sources de la crise en incriminant le pouvoir en place et en remettant en cause les fondements de l'idéologie étatique, quitte à malmener sérieusement le mythe du FLN libérateur en exhumant toutes ses exactions. La plus emblématique d'entre elles est sans doute le massacre des messalistes de Melouza, auquel Anouar Benmalek consacre significativement un roman entier (Le Rapt 2009). L'emploi de la langue française en pareil cas

\footnotetext{
1 On s'accorde à dénombrer au moins 100000 morts, auxquels s'ajoutent les victimes de massacres résiduels au début des années 2000 (Ménard 2003, 6).
} 
fait peser sur les auteurs un soupçon de traitrise et de racolage. Que l'on considère le texte que Yasmina Khadra attribue à l'un des personnages de Qu'attendent les singes: Ed Dayem, excédé par le succès parisien d'un jeune romancier francophone fictif, Baasous Llaz, présenté de manière extrêmement négative :

Selon Malek Bennabi, il y a le colonisé et il y a le colonisable. Les colonisés aspirent à se soustraire au joug qui les assujettit; les colonisables, même libres, ont constamment besoin d'un maître. Certains se bradent sur la place de Paris et, ne trouvant pas preneurs, ils s'acharnent sur ceux qui réussissent. D'autres s'engouffrent allègrement dans les lobbies hostiles à ce qui est beau et grand chez nous et contribuent, avec un rare dévouement, à défigurer l'image de nos justes en espérant se donner une visibilité. Ils seront acclamés, distingués, vantés, puis forcés de dire, en signe de reconnaissance servile, exactement ce que leur maîtres veulent entendre car il n'est plus savoureuse victoire pour nos ennemis que de voir notre pays traîné dans la boue par ses propres rejetons. (Souligné dans le texte) (Khadra 2014, 82).

On notera que le cas de Yasmina Khadra est particulièrement représentatif de cette problématique qui est d'ailleurs constamment explorée dans ses œuvres. Son succès critique et commercial retentissant en France, il le doit en partie à des fictions romanesques où la critique de la société algérienne est omniprésente. Toutefois, quand dans des entretiens avec les médias français il est invité à approfondir cette critique, il émet d'importantes réserves, au nom d'une loyauté nationale bien compréhensible. Il y a alors rupture entre les dénonciations de l'auteur et les discours de l'homme public. Se trouve traduite toute la complexité du positionnement des auteurs algériens francophones.

S'ajoute à ce soupçon l'extrême opacité de la guerre civile algérienne dont on n'a toujours pas identifié avec certitude les véritables acteurs et qui ferait dire avec Balzac que «la hardiesse du vrai s'élève à des combinaisons interdites à l'art tant elles sont invraisemblables et peu décentes » $(1973,176)$ si les écrivains de la décennie noire n'avaient pas pourtant sondé sans relâche les ténèbres d'une réalité « obscure et insaisissable » (Mahiou 2001, 14). On retient actuellement trois hypothèses pour désigner les véritables responsables : les « seuls islamistes », « des groupes liés au pouvoir ou manipulés par lui » ou des « responsabilités imputables aux uns et aux autres» (Mahiou 2001, 14-15). Se prononcer et proposer une réponse à la fameuse question «qui tue qui ? » (Charef 1998, 5) équivaut nécessairement à diviser les lecteurs.

\section{b) La réception française}

En France, on peut dans un premier temps évoquer une certaine réticence des milieux universitaires en raison d'un retour au réalisme jugé comme un abandon des explorations formelles de la génération des « enfants terribles» (Bonn 1999, 11). Il y aurait ensuite le problème posé par la 
réception du lecteur «lambda $»^{2}$, dont l'engouement pour la production littéraire algérienne de ces années sombres a pu s'apparenter, si ce n'est au "voyeurisme » auquel Charles Bonn $(1999,16)$ fait référence, à des attentes documentaires alimentées par les stratégies éditoriales françaises qui mettent en avant «le contexte politique duquel le texte est présenté comme le reflet fidèle, à travers un quotidien le moins distancié possible » (Bonn 1999, 17).

On peut avancer, d'autre part, une certaine tendance à la récupération politique dont les motivations sont troubles et que Yasmina Khadra dénonce dans La Part du mort à travers le portrait du professeur Allouche :

Très vite, notre éminent savant s'aperçut que les égards de ses confrères occidentaux n'étaient que des pièges succulents, que les prix qu'on lui décernait avaient un arrière-goût d'à-valoir, et que ses travaux d'érudits se découvraient des accents politiques puisqu'il passait plus de temps à hanter les salles de rédaction et les salons des ONG que les ateliers universitaires. On ne l'applaudissait plus pour ses recherches; on saluait ses prises de position contre la dictature qui sévissait au bled. $(2004,30)$.

Certes, il s'agit là d'un psychanalyste et non d'un romancier, mais la transposition paraît tout à fait envisageable.

L'enthousiasme douteux avec lequel ont été reçues les fictions référentielles critiques de la décennie noire trouverait enfin son exact opposé chez certains intellectuels de gauche, qui ont craint que leur souscription à une dénonciation conjointe de l'intégrisme religieux et de l'État algérien ne s'apparente au fond à une manière de ressentiment historique. Sur ce point, le témoignage de l'écrivain tunisien Albert Memmi sur les difficultés qu'il a rencontrées lors de la promotion de son Portrait du décolonisé arabo-musulman est éclairant :

Je m'attendais à quelque émotion du côté des ex-colonisés, particulièrement des Arabo-musulmans. Or mes lecteurs ex-colonisés et leurs descendants ne furent apparemment pas scandalisés, pas même surpris par mon entreprise. Au contraire, ce fut comme s'ils l'attendaient ; [...] Non, curieusement mes soucis vinrent d'ailleurs. Je mentirais en disant que je ne m'y attendais pas du tout ; ce n'était qu'une confirmation, elle n'en fut pas moins amère. Je passe sur quelques pitoyables palinodies. Une semaine après la parution du livre, je reçois une invitation à passer sur l'antenne de Radio Libertaire. La veille, nouveau coup de téléphone: il n'y aura pas d'émission. Pourquoi ? "Vous dites des choses insupportables à nos auditeurs. - Peut-être; mais laissez-moi m'en expliquer !" Non, c'est définitivement non. (2004, 217-218).

Et la situation de se rejouer, d'après Albert Memmi, avec Libération. Difficile ici de ne pas penser à la mise au point de Pascal Bruckner, que ces questions ont spécialement préoccupé : «la critique de tel ou tel régime n’est pas l'apologie secrète du colonialisme et l'opposition de l'ami qui ne fait pas chorus est plus précieuse que les insultes de l’ennemi déclaré !» (1983, 247).

2 Pour reprendre le terme utilisé par Boualem Sansal dans un entretien filmé avec un écrivain franco-algérien (Amellal 2014, 10'06"). 
Plus spécifiquement, les intellectuels occidentaux ont été choqués par l'interruption du processus électoral après la victoire du FIS au premier tour des législatives de 1991, comme l'explique ici Rachid Mimouni: «Les Occidentaux tiennent à rappeler que le FIS a gagné les élections du 26 décembre. Les intégristes de la démocratie rejoignent en de nombreux points ceux de l'islam. Les uns et les autres se crispent sur des principes dont ils exigent l'application littérale.»(1992, 152). Naturellement, Mimouni rêvait d'une démocratie algérienne, et l'on sait comme la guerre civile l'a blessé. Mais justement, comment dénoncer, au cœur de l'urgence, l'islamisme meurtrier du conflit si une partie du lectorat lui trouve implicitement des circonstances atténuantes?

Cet état des lieux nous a paru indispensable pour mieux comprendre pourquoi va s'imposer progressivement chez les auteurs algériens francophones, passée la sidération des premières années du conflit, une théorisation de la relation au lecteur et la mise en place d'un nouveau contrat de réception.

\section{Réécrire le réel}

Boualem Sansal, haut fonctionnaire, ingénieur et économiste, fait partie de ceux que la guerre civile a jetés dans l'écriture. Son premier roman, Le Serment des barbares ${ }^{3}$, parait en 1999 au terme d'une rédaction s'étalant sur trois années. L'auteur choisit d'y « rendre la confusion par la confusion » (Amellal 2014, 09'00") et aboutit à une œuvre dense, intergénérique et à l'énonciation innovante.

Comme YB dans L'Explication, "polar mystico-politique» (Chollet 1999, \$4) édité la même année, Sansal brouille les repères et entremêle au discours et aux données référentielles brutes la fiction la plus débridée. Si les moyens diffèrent, la démonstration semble commune aux deux auteurs : il s'agit avant toute chose de remettre en cause la fiabilité des médias d'expression du réel que sont le journalisme et les sciences humaines, et tout particulièrement l'histoire, dans un pays où le référent est insaisissable, falsifié par l'idéologie et où la notion de réalité est donc largement remise en question.

Il n'est pas anodin que ces deux romans, tardifs dans la décennie noire, s'appuient sur le même type de réflexion formelle : les principaux écueils qui entravent la réception des romans algériens francophones et du discours qu'ils véhiculent sont connus de leurs auteurs, qui tentent clairement de les contourner. Ce travail est très net chez Boualem Sansal, qui va tenter de palier le problème dans Le Serment des barbares en impliquant le lecteur de façon à ce qu'il ne puisse pas recevoir le roman de manière passive.

\footnotetext{
${ }^{3}$ Sansal, Boualem. Le Serment des barbares. Paris : Gallimard, 1999. Dans l'article, les citations tirées de ce roman seront suivies du titre abrévié $(S B)$ et du numéro de la page.
} 


\section{Déjouer l'attente documentaire}

Ainsi, pour combattre l'attente documentaire du lectorat occidental, l'auteur refuse tout didactisme: pas de notes de bas de page ou de glossaires comme on trouve chez Yasmina Khadra ou Rachid Mimouni, mais des données référentielles non explicitées, des insinuations diffuses qui imposent au lecteur étranger, voire algérien, un travail de recherche sans lequel le texte est inintelligible. Ainsi : «Mon œil ! Le FIS est une invention des Kabyles pour se libérer des Arabes. Serait-ce le remake du complot berbériste de 49, le complot des marabouts? Comment réagira l'armée ? Va-t-elle sauter le pas et se débarrasser des civils ? » $(S B, 199)$.

Comme on le voit ici, le texte tend à accueillir sans signalement ni distribution toutes les voix de la rue algérienne et toutes les rumeurs qu'elles diffusent, sans spécifier le degré de vraisemblance dont on peut les créditer. Ainsi, si la création du FIS par les Kabyles parait hautement improbable, la question de l'implication de l'armée dans certaines tueries, celle de Bentalha notamment, s'est réellement posée, bien qu'elle soit encore à ce jour irrésolue. La rumeur acquiert un statut ambivalent; parce que les médias traditionnels d'expression du réel sont peu sûrs, elle est une possible source alternative pour qui entend rétablir la vérité des faits, à condition d'être prudent. Son introduction sur la page est en tout cas emblématique d'un roman où l'on ne peut ni tout accepter, ni tout rejeter : l'ensemble des informations référentielles délivrées par le texte demande à être étudié et approfondi.

Le tout est relayé par une prose complexe qui rend impossible toute lecture en diagonale et sollicite un permanent exercice de la mémoire, dans la mesure où l'auteur s'ingénie à multiplier les ambigüités syntaxiques. Qu'on en juge en considérant ici le groupe nominal «le bâtiment» (ligne 1) et les reprises anaphoriques dont il est l'antécédent (que nous mettrons en italique) :

À moins d'être un coquin, on ne peut réussir dans le bâtiment. Dans la profession, on s'emploie à passer pour des victimes de l'ordre mondial, ce qui suppose une totale méconnaissance de ce qu'est l'ordre chez soi. On déploie une telle autre variété d'efforts qui, s'ils étaient mis au service de la cause, rendraient le logement abondant et à la portée des nomades tout en laissant un gentil profit à ces méchantes gens. Ainsi n'est pas, le système tourne à l'envers et ses hommes n'ont pas le sens du bien. Le regard innocent n'entrave que pouic. Son remue-ménage est indescriptible et sa finalité obscure : voyez : il exploite près de deux millions de bras, avale bon an mal an six à sept millions de tonnes de ciment, autant de fer et de bois et une quantité invraisemblable de clous ; $(S B, 41-42)$.

C'est le sens qui permet finalement l'identification de l'antécédent, placé en concurrence avec deux autres substantifs masculins au singulier qui le séparent de ses reprises anaphoriques. Si l'on ajoute à cela le jeu des personnifications, on obtient un texte qui exige une concentration permanente et de fréquents retours en arrière. Se confronter au Serment des barbares, c'est s'interdire d'aller « droit aux articulations de l'anecdote » (Barthes 1973, 22). 


\section{Le refus du voyeurisme}

Le lecteur-voyeur avide de descriptions de tueries sanguinaires est également invité à passer son chemin :

Passons sur les atrocités: les tangos égorgent ce qu'ils trouvent sur leur chemin, chauffeurs en livrée, gardes endormis, servantes en sueur, bébés mis au frais sous les arbres, enfoncent les portes, font irruption dans la grande cour, illuminée a giorno. Inutile de s'appesantir sur le désarroi des convives, les mouvements de panique vite réprimés, ni sur le comportement des sauvages. Tout se passe comme à l'accoutumée; les hommes sont égorgés, les femmes éventrées, les bébés fracassés contre les murs, etc. (SB, 221).

D'évidence, la prétérition féroce du narrateur rejette une forme de banalisation impliquée par la réécriture des mêmes séquences macabres devenues autant de «stéréotypes » (Haraoui-Ghebalou 1999, 53) attendus et qui perdent donc leur pouvoir de dénonciation. Précisons que la description du massacre que nous reproduisons ici se trouve de surcroît intégrée à un récit isolé du reste du roman par un changement de typographie, de temps grammaticaux, en rupture nette avec l'intrigue principale. Ce bref intermède, intitulé "Histoire de mariage ", est mystérieux jusque dans son énonciation puisqu'il se clôt de la sorte: "Merci cher Ali, de ton témoignage qui n'en est pas vraiment un.» $(S B, 222)$. Or, on ne retrouve cet Ali nulle part dans le corps du roman.

Le «témoignage » d'Ali relate en fait les noces sanglantes de la fille du grossiste Si Mourad, au cours desquelles une katiba du GIA a fait irruption et massacré tous les convives. Pourtant, étrangement, le père de la mariée se comporte le lendemain comme si rien ne s'était passé et on découvre plus tard que les « huiles » présentes au mariage sont sorties parfaitement indemnes de la tuerie. Nous partageons à ce propos l'interprétation d'Estelle Maleski qui voit dans «Histoire de mariage » une parabole semblant « inviter le lecteur à la méfiance : de même que tout enquêteur confronté à l'inconnu - non su ou tu -, le lecteur se doit d'être prudent» $(2003,342)$.

En somme, l'auteur illustre la confusion qui s'est instaurée entre la réalité du terrorisme et la légende qui se crée simultanément autour de cette réalité, et qui d'après lui a tout intérêt à être alimentée par le pouvoir en place pour justifier le maintien d'une politique répressive et autoritaire. De fait, la fascination du lecteur occidental pour les tableaux d'horreur a des conséquences en ce qu'elle conforte indirectement une politique d'instrumentalisation trouble du terrorisme : si l'on part du principe que l'offre s'ajuste à la demande, elle occasionne une surenchère de descriptions macabres dans la littérature et les médias. Il est ainsi tout à fait signifiant que la seule description macabre soit placée en marge de la trame romanesque, manière symbolique d'en rejeter la pratique.

\section{L'indicible islamiste}

Dans Le Serment des barbares, Boualem Sansal met au point un régime narratif mixte que l'on peut qualifier de semi-omniscient. Le narrateur, de 
prime abord un narrateur omniscient utilisant la troisième personne du singulier, est systématiquement externe quand le récit se confronte à la représentation des terroristes islamistes. Il n'est pas question ici de leur donner la parole comme le fait par exemple Anouar Benmalek dans Les Amants désunis (1998), qui entend offrir dans son roman une tribune à chaque acteur de la crise algérienne. Il en est ainsi de ce personnage, membre du groupe armé qui a capturé l'héroïne :

Nous ne sommes pas des assassins mais des exécutants. Ne les plains pas, ces trois-là. Tous les trois font partie des milices du pouvoir! Au moins eux ont bénéficié d'un couteau. C'est propre, net et miséricordieux, le couteau! Mon père, lui, a été torturé par l'armée au chalumeau. Moi j'étais un commerçant aisé, j’avais tout, mais je n'ai plus été capable de supporter la hogra, le mépris, les passe-droits, la débauche des femmes, l'insulte faite à la religion par ceux qui nous gouvernent. (SB, 299).

La conviction politico-religieuse n'est pas absente du discours de ce terroriste. Cependant, il est clair que le déclencheur de son passage à la violence armée est un traumatisme personnel, et le désir de vengeance qu'il entraîne. Dans ce cas, l'empathie est possible. Rien de tel chez Sansal, qui se refuse à humaniser ceux qu'il appelle « les fous d'Allah » $(S B, 270)$ et à courir le risque que le lecteur ne leur trouve des circonstances atténuantes. Les terroristes islamistes sont sous le coup d'une impossible mimésis, chimères monstrueuses dont la représentation se colore systématiquement d'une mythification aux accents grotesques: ce sont des «djinns peinturlurés sautillant sur les sentiers de la guerre » $(S B, 87)$, des « sortes de hérauts portant barbe-claquettes-gandoura, la panoplie bcbg de la terreur» $(S B, 303)$, qui ne dépareraient pas dans quelque bestiaire médiéval fabuleux : «Au demeurant, l'oiseau n'était pas quelconque ; il avait une tête ! une tête d'islamiste ; un crâne d'œuf mal rasé, le front simiesque, la mandibule prognathe, le nez en bec d'aigle, un bouc miteux et des yeux de serpent frit. » (SB, 370).

Les intégristes ne recouvrent leur humanité que dans la mort, et le héros du roman, le vieil inspecteur Larbi, en fait la douloureuse expérience :

Le policier se pencha sur les dépouilles. Un spasme le secoua. Les hommes y virent une réaction naturelle devant la boucherie. Le vieil homme était horrifié par d'autres choses contre lesquelles on ne peut rien quand elles vont ensemble: la bêtise des intégrismes, la naïveté des jeunes et l'avidité des mercantis au pouvoir. Trois des huit morts étaient des gosses, ou pas loin, et les cinq autres pouvaient très bien être de vieux copains de la JFLN. (SB, 254).

Ce retour parmi les vivants qui ne s'effectue paradoxalement que par la mort exprime la fracture ontologique totale qui sépare le narrateur et son héros, avec lequel il est en symbiose, des terroristes, irréductibles à la littérature et à ses procédés, parmi lesquels la focalisation interne. 


\section{La narration mise à l'épreuve}

L'instance narrative du Serment des barbares, confrontée à l'indicible de l'islamisme, est en revanche capable d'accéder à la psyché des personnages ordinaires, adjuvants ou petits escrocs véreux et sexistes, sans aucune démarcation stylistique. C'est d'autant plus troublant que d'imperceptibles basculements en focalisation interne sont utilisés tout au long du roman pour se connecter aux pensées de Larbi, quasiment indissociable du narrateur.

Le lecteur identifie et surmonte rapidement la difficulté énonciative, l'interprétant comme le signal de valeurs partagées par le narrateur et son héros. Il est donc extrêmement surprenant que cet arasement stylistique soit aussi à l'œuvre lorsque la focalisation interne permet le contact avec des personnages négativement crédités. C'est une manière de piéger le lecteur et de l'inciter à la prudence face à tout texte qui entend prendre en charge une réalité complexe : bercé par la voix narrative et emporté par une lecture rapide, va-t-il s'apercevoir que le discours est devenu inadmissible?

Il faut noter que dans les romans qui suivent Le Serment des barbares, Boualem Sansal épure l'énonciation en privilégiant la première personne mais radicalise dans le même temps la mise à mal du lecteur. Précisément parce que la première personne accentue l'illusion de vérité ${ }^{4}$ et suscite l'adhésion spontanée, l'auteur met au point un vraisemblable narratif original.

Il ne s'agit pas, comme on peut le voir dans 41, rue de l'Aigle d'Abdelkader Djemaï (2000), de jouer sur un sentiment d'identification trouble en donnant la parole à un monstre, mais de mettre au point une figure narrative globalement sympathique, relai fréquent des idées que Sansal expose dans les divers entretiens que les critiques et journalistes lui accordent, et pourtant susceptible d'erreurs, d'emportements, de propos misogynes ou xénophobes qui ne sont en aucun cas attribuables à l'auteur. Là encore le but est d'inciter ceux qui se confrontent à des textes référentiels et polémiques à prendre conscience que tout scripteur est faillible, et qu'une lecture responsable ne peut faire l'économie d'une distance critique et d'une entreprise de recherche individuelle qui doit accompagner l'émergence d'une saisie personnelle, documentée et sans manichéisme.

Et Boualem Sansal de mettre à l'épreuve sans relâche ce pacte de lecture implicite en abordant tous les sujets que des crispations trop fortes ont fini par transformer en constructions théoriques abstraites, figées dans des positions antagonistes unilatérales. Attention toutefois, il ne s'agit pas pour l'auteur de se dédouaner de ses prises de position en se réfugiant au besoin derrière l'argument de littérarité. Ce qu'il propose est plus simplement une nouvelle façon de recevoir les textes. Idéalement, le lecteur sansalien ne doit plus réagir de manière épidermique et buter sur des tabous: tout peut être abordé, et « tout est critiquable » (Adler 2015, 30'53").

\footnotetext{
4 C'est du moins ce que postule Käte Hamburger pour laquelle « c'est bien la première personne qui confère aux énoncés les plus improbables une apparence de réalité » (cité par Schmitt 2010, 24).
} 


\section{Le recours au mythe}

Il est généralement établi pour un lecteur contemporain qu'une représentation mythique ne se reçoit pas telle quelle parce qu'elle brasse indifféremment vérités, fantasmes et croyances. Les éléments de réel que propage le mythe sont constitutivement cryptés par le recours à la fable et exigent en ce sens une approche herméneutique, et non littérale. Ces précisions une fois données, il parait difficile de recevoir de manière brute le mythe de l'âge d'or colonial avec lequel joue très nettement Boualem Sansal :

En ce temps, Rouiba vivait la quiétude, l'opulence; elle était pimpante, coquette, sensuelle et aimait à se parer de fleurs ; le laurier avait sa préférence ; il en a profité pour se multiplier, le simplet, et prospérer même là où sa présence ne pouvait amener que des soucis. [...] Couchée dans cette fabuleuse plaine de la Mitidja, grosse toute l'année, elle déversait sur la capitale, à pleins camions, les fruits de ses entrailles et de son labeur. (SB, 10-11).

Rien ne manque : l'abondance, la douceur de vivre, la paix. On pourrait dire avec Ovide qu'à Rouiba: «Le printemps était éternel, les tranquilles zéphyrs caressaient de leur souffle tiède les fleurs nées sans semence » (1966, 44) même si, à la différence de la retranscription ovidienne du mythe, le «labeur» n'est pas absent. En personnifiant la ville, l'auteur parvient malgré tout à réactualiser astucieusement le mythe puisque aucune intervention humaine n'est évoquée. Le problème ne se pose même pas si l'on considère l'âge d'or tel qu'il apparaît dans la Genèse : «L'Éternel Dieu prit l'homme et le plaça dans le jardin d'Éden pour le cultiver et le garder. » (Gn, 2-15). Contrairement à la tradition gréco-latine, le travail ne marque pas la fin de l'âge d'or, bien au contraire.

En insistant sur la prospérité de la terre et en précisant, à propos de Rouiba, que «Le travail était pourtant sa religion et son unique souci. » (SB, 11), Sansal conserve l'idée biblique du statut de gardien et de cultivateur confié à l'homme. L'une des conséquences de la Chute, c'est la fin de l'abondance et la difficulté à cultiver un sol devenu pauvre et envahi par la mauvaise herbe. C'est ce qui se produit à Rouiba, comme le montre cette représentation syncrétique où la fin du labeur heureux en Eden et des richesses qu'il prodiguait s'associe à un véritable âge de fer :

Les complexes industriels, tels des chars d'assaut, ont envahi les domaines des colons; les ateliers privés, rémoras des grands combinats publics, se sont infiltrés dans les chais des viticulteurs et les caves des distillateurs; et, dans la foulée du déclin programmée de l'agriculture, les halles aux fruits et légumes qui pullulaient autour de la ville et embaumaient sa tiède et suave atmosphère furent investies et transformées en entrepôts d'État. Des bardages métalliques et des dispositifs tatillons sont venus les protéger de toute atteinte. $(S B, 13)$. 
Le prologue virtuose du Serment des barbares, saturé par le mythe et le réalisme merveilleux ${ }^{5}$, devrait d'emblée inviter à la prudence. Le fait qu'il ait été reproché à l'auteur de plaider en faveur de la colonisation et de diffuser une «nostalgie malsaine » (Mouffok 1999, 13) de la période coloniale est en ce sens révélateur à la fois des crispations de la réception, et de la nécessité d'un travail littéraire qui les anticipe et les théorise.

Si pareil traitement de la période coloniale constitue une réponse radicalement transgressive à l'histoire officielle, Boualem Sansal n'est pourtant pas le seul auteur algérien à avoir éprouvé l'urgence de poser un regard pacifié sur le passé : les entreprises fleurissent aujourd'hui qui entendent démontrer qu'il y eut avant l'indépendance des liens entre les différentes communautés, des amitiés nouées, une mémoire en commun et l'amour d'une même terre ${ }^{6}$. Sonder cette mémoire devient vital au cours des années de guerre civile où s'impose l'idée qu' «À un homme qui a mal à son passé, on peut proposer n'importe quel avenir pourvu qu'il y ait la nostalgie et un plan de ressourcement à la clé. » (SB, 437).

La réécriture de l'âge d'or dans Le Serment des barbares fonctionne comme une incitation puissante: le mythe permet d'introduire dans le roman référentiel une masse d'informations dissidentes mais avertit dans le même temps qu'il va s'agir d'une parole fantasmée et idéalisée, dont on ne peut éprouver et extraire la vérité première qu'en la confrontant à d'autres sources. Le lecteur est invité à mener une enquête qui lui permette à son tour de mettre à l'épreuve la vision manichéenne de l'histoire qu'a imposée le FLN et de tirer ses propres conclusions.

\section{Un combat à mener ensemble}

Par l'ensemble de ces procédés Boualem Sansal refuse un statut surplombant et moralisateur, écarte la tentation de substituer avec autorité sa propre conception de la vérité à la doxa qu'il rejette et compte sur cette intégrité pour contrer les mensonges et les falsifications qui dénaturent l'histoire algérienne au motif que: «L'histoire n'est pas l'histoire quand les criminels fabriquent son encre et se passent la plume. Elle est la chronique de leurs alibis et ceux qui la lisent sans se brûler les yeux sont de faux témoins. » (SB, 461).

Toute l'œuvre de l'auteur va dans ce sens : combattre les mensonges qui sont d'après lui à l'origine de la violence qui s'est déchaînée, laquelle peut ressurgir à tout moment si une réconciliation de l'Algérie avec son passé n'a

\footnotetext{
5 Au sens où le définit Charles Scheel : « fondre un code réaliste et un code merveilleux par le biais d'une poétisation presque constante de la narration qui exprime l'exaltation de l'auteur par rapport à son thème et à son écriture » $(2005,202)$.

${ }^{6}$ Cf. Lucienne Martini : « un retour sur la mémoire de la période coloniale s'amorce et dans les textes de ces dernières années, il semble que le regard soit en train de changer, que cette période puisse apparaitre sous des couleurs moins sombres et que son héritage puisse être reconnu aussi, dans des aspects positifs constitutifs d'une identité algérienne autonome et authentique » $(2001,354)$.
} 
pas lieu, et que, comme le dit Yasmina Khadra, «l'Histoire [reste] le pire ennemi de l'Avenir» $(2004,120)$. Mais ce n'est pas un combat que Boualem Sansal entend livrer seul, et chacun est encouragé ${ }^{7}$ à y apporter sa contribution. Tous en retour s'en trouvent enrichis, tant la réflexion sansalienne s'universalise en une véritable éthique de lecture.

\section{Bibliographie}

Amellal, Karim. «Entretien de l'écrivain Boualem Sansal avec Karim Amellal ». [En ligne]. Mis en ligne le 10 décembre 2014.URL: https://www.youtube.com /watch?v=doyO-i9rhPA. (Consulté le 8 juin 2015).

Adler, Laure. "Hors champs ». France culture. [En ligne]. Mis en ligne le 02 février 2015. URL: http://www.franceculture.fr/emission-hors-champs-boualemsansal-2015-02-02. (Consulté le 03 février 2015) : 30'53".

Balzac de, Honoré. Splendeurs et misères des courtisanes. Paris : Gallimard, 1973 [18381847].

Barthes, Roland. Le Plaisir du texte. Paris : Seuil, 1973.

Benmalek, Anouar. Les Amants désunis. Paris : Calmann-Levy, 1998.

Benmalek, Anouar. Le Rapt. Paris : Fayard, 2009.

Bonn, Charles. «Introduction». In: Bonn, Charles et Farida Boualit (dir.). Paysages littéraires algériens des années 90 : témoigner d'une tragédie?. Paris: L'Harmattan, $1999: 8-24$.

Boualit, Farida. "La littérature algérienne des années 1990: témoigner d'une tragédie ?». In : Bonn, Charles et Farida Boualit (dir.). Paysages littéraires algériens des années 90 : témoigner d'une tragédie ?. Paris: L'Harmattan, 1999 : 25-40.

Bruckner, Pascal. Le Sanglot de l'homme blanc. Paris : Seuil, 1983.

Charef, Abed. Algérie : autopsie d'un massacre. Paris : L'Aube, 1998.

Chollet, Mona. «YB, journaliste et écrivain - L'Intransigeant d'Alger ». Périphéries. [En ligne]. Mis en ligne le 13 août 1999. URL: http://www.peripheries.net /article197.html. (Consulté le 13 juin 2015).

Djemaï, Abdelkader. 31, rue de l'Aigle. Paris : Gallimard, 2000.

Haraoui-Ghebalou, Yamilé. «Litanies mortuaires et parcours d'identité ». In : Bonn, Charles et Farida Boualit (dir.). Paysages littéraires algériens des années 90 : témoigner d'une tragédie?. Paris : L'Harmattan, 1999 : 53-59.

Khadra, Yasmina. La Part du mort. Paris : Gallimard, 2004.

Khadra, Yasmina. Qu'attendent les singes. Paris : Julliard, 2014.

Mahiou, Ahmed. "Les contraintes et incertitudes du système politique». In: Jean-Robert, Henry et Ahmed Mahiou (dir.). Où va l'Algérie? Paris : Karthala-Ireman, $2001:$ 13-34.

Maleski, Estelle. Le Roman policier à l'épreuve des littératures francophones des Antilles et du Maghreb: enjeux critiques et esthétiques. Bordeaux: Université Michel de Montaigne-Bordeaux III, thèse de doctorat réalisée sous la direction de Mme Martine Job, 2003.

\footnotetext{
${ }^{7}$ Nous savons que Boualem Sansal dit prendre en compte lorsqu'il écrit au moins deux lectorats, algérien et français (Amellal 2014, 7'35").
} 
Martini, Lucienne. "Littérature et travail de mémoire ». In : Jean-Robert, Henry et Ahmed Mahiou (dir.). Où va l'Algérie ?. Paris : Karthala-Ireman, 2001 : 347-363.

Memmi, Albert. Portrait du décolonisé arobo-musulman et de quelques autres. Paris: Gallimard, 2004.

Ménard, Robert. «Préface ». In : Algérie, le livre noir. Paris : La Découverte, 2003 : 5-8.

Mimouni, Rachid. De la barbarie en général et de l'intégrisme en particulier. Paris : Éditions Le Pré Aux Clercs, 1992.

Mouffok, Ghania. «De Boudjedra à Sansal. Le malaise des barbares ». La Tribune. 2223 octobre 1999.

Moura, Jean-Marc. Littératures francophones et théorie postcoloniale. Paris : PUF, 1999.

Ovide. Les Métamorphoses [Trad. Joseph Chamonard]. Paris : Garnier, 1966.

Sansal, Boualem. Le Serment des barbares. Paris : Gallimard, 1999.

Scheel, Charles. W. Réalisme magique et réalisme merveilleux: des théories aux poétiques. Paris : L'Harmattan, 2005.

Schmitt Arnaud. Je réel/Je fictif-Au-delà d'une confusion postmoderne. Toulouse : Presses Universitaires du Mirail, 2010.

YB. L'Explication. Paris : Lattès, 1999. 